\title{
Correction to: 12. Deutscher Wirbelsäulenkongress Jahrestagung der Deutschen Wirbelsäulengesellschaft, 30. November - 2. Dezember 2017, Stuttgart
}

๑) Springer-Verlag GmbH Germany, part of Springer Nature 2018

\section{Correction to: Abstracts for 12th German Spine Conference-Annual Meeting of the German Spine Society Eur Spine J (2017) 26:2978-3057 https://doi.org/10.1007/s00586-017-5336-8}

Unfortunately, the abstract from V52 to V58 have been missed out in the original publication and they are given as below.

\section{52}

Surgical site infections following instrumented stabilization of the spine: an evaluation of 138 clinical cases

*U. Dapunt ${ }^{1}$, C. Bürkle ${ }^{1}$, F. Günther ${ }^{2}$, W. Pepke ${ }^{2}$, S. Hemmer $^{1}$, M. Akbar ${ }^{1}$

${ }^{1}$ Universitätsklinikum Heidelberg, Klinik für Orthopädie und Unfallchirurgie, Heidelberg, Deutschland

${ }^{2}$ Universitätsklinikum Heidelberg, Zentrum für Infektiologie, Heidelberg, Deutschland

Bacterial biofilm infections are still a feared complication in the field of orthopaedics. Bacteria attach to the implant surface and embed themselves in a slimy matrix: the socalled "biofilm": In this form, bacteria are better protected and more resistent towards antibiotics and biocides. There is an abundance of literature available on prosthetic joint infections of the hip and knee. Therefore, therapeutic guidelines of implant associated infections have been mostly derived from these data.

Aim of this study was to evaluate a high number of implant associated infections following instrumented stabilization of the spine concerning detected bacteria und the course of the disease. Additionally, the results were compared to prosthetic joint infections of the hip and knee.

We performed a retrospective single-center analysis of implant-associated infections of the spine. From 2010-2014 a total of 138 patients were included in the study. The following parameters were evaluated: CRP serum concentration, microbiological evaluation of tissue samples, the time course of the disease, indication for instrumented stabilization of the spine, localization of the infection, and the number of revision surgeries required until cessation of symptoms.

Coagulse-negative Staphylococcus spp. were most commonly detected, followed by faecal bacteria. In $23.2 \%$ of cases, no bacteria were detected despite clinical suspicion of an infection. Most patients suffered from degenerative spine disorders (44.9\%), followed by spinal fractures $(23.9 \%)$, non-degenerative scoliosis $(20.3 \%)$ and spinal tumors (10.1\%). Surgical site infections occurred predominately within 3 months $(64.5 \%)$, late infections after 2 years were rare $(4.3 \%)$, in particular when compared to prosthetic joint infections. Most cases were successfully treated after one revision surgery (60.9\%), but there were significant differences between bacteria species. Faecal bacteria were more difficult to treat and often required more than one revision surgery.

In summary, we were able to demonstrate significant differences between spinal implant-associated infections and prosthetic joint infections. These aspects should be considered early on in the treatment of surgical site infections following instrumented stabilization of the spine. 


\section{53}

Surgical Training in Spine Surgery: Safety and Patientrated Outcome

${ }^{*}$ G. Waisbrod ${ }^{1}$, T. Fekete ${ }^{2}$, A. F. Mannion ${ }^{2}$, F. Kleinstück ${ }^{2}$, F. Porchet ${ }^{2}$, D. Jeszenszky ${ }^{2}$, D. Haschtmann ${ }^{2}$

${ }^{1}$ Schweizer Paraplegiker Zentrum Nottwil, Wirbelsäulenchirurgie, Nottwil, Schweiz

${ }^{2}$ Schulthess Klinik, Wirbelsäulenchirurgie, Zürich, Schweiz

Background: Surgical education of spine surgery residents (RES) in the operation theatre is a decisive part of their training. However, patient safety and optimal outcome must have the greatest priority and should not be impaired on behalf of the education. Balancing these two aspects might become a dilemma and may result theoretically in increased morbidity and less satisfied patients. The aim of this study was to investigate the difference between procedure carried out either by experienced spine surgeons or by spine surgery residents under supervision on surgical complications and patient reported outcomes in patients undergoing lumbar procedures.

Methods: This was a single-center retrospective analysis of prospectively collected data within the EUROSPINE Spine Tango Registry, using data from our institution between the years 2004-2015. The study included a total of 1413 patients. Patients operated on by board certified spine surgeons (BCS) served as control group. Patients were divided into three groups based on the surgical procedure they underwent: lumbar single level fusion (SLF): RES $n=60$, BCS $n=261$, single level decompression for lumbar spinal stenosis (SLD): RES $n=72$, BCS $n=245$ and surgery addressing disc hernia (DH): RES $n=247$, BCS $n=528$. Patients completed the multidimensional Core Outcome Measures Index (COMI; 0-10 scale) preoperatively, and 3 and 12 months postoperatively. Multiple linear regression models were used to investigate the influence of resident performance on the outcomes of interest.

Results: There were no differences in surgical or medical complication rates $(\mathrm{p}>0.05)$. Blood loss was found to be significantly higher in resident performance only for SLD $(p<0.05)$ and operation time was significantly longer only in DH cases $(p<0.05)$. Length of hospitalization was slightly but not significantly higher in SLF cases $(\mathrm{p}=0.178)$. Patient outcome reflected by COMI scores was similar preoperatively for RES and BCS. Scores significantly improved after all three types of procedures $(\mathrm{p}<0.0001)$ without difference between teaching cases and control group ( $p>0.05)$ Conclusion: Surgical training of spine surgery residents under guided supervision by board certified spine surgeons is safe as it is not associated with increased morbidity and mortality. Furthermore, patient reported outcome revealed improvement after all three procedure types and is not affected by the teaching cases.
V 54

CT-/MRI-based analysis of the chronological progress of bilateral insufficiency fractures of the sacrum in geriatric patients

*T. Mendel ${ }^{1}$, B. W. Ullrich ${ }^{1}$, P. Schenk ${ }^{2}$, G. O. Hofmann ${ }^{1,3}$, *F. Klauke ${ }^{1}$

${ }^{1}$ BG Klinikum Bergmannstrost Halle, Klinik für Unfall- und Wiederherstellungschirurgie, Halle (Saale), Deutschland

${ }^{2}$ BG Klinikum Bergmannstrost Halle, Stabsstelle Forschung, Halle (Saale), Deutschland

${ }^{3}$ Universitätsklinikum Jena, Klinik für Unfall-, Hand- und Wiederherstellungschirurgie, Jena, Deutschland

Introduction: Bilateral insufficiency fractures of the sacrum (BIFS) represent an increasing disease entity in geriatric patients and are frequently associated with pain-related immobility. In opposite to traumatic injuries of the sacrum, which are well understood, pathomechanism as well as the underlying chronological progress of BIFS induced by osteoporotic bone rarefication contains several outstanding questions.

Materials and methods: In a 3-years period (2014 to 2016) 273 geriatric patients ( $>65$ years) with pelvic injuries were treated in our hospital. In 64 cases (23.4\%) a bilateral insufficiency fracture of the sacrum was diagnosed. In 38 of these patients ( $\varnothing 77,8$ years, male: 3 ; female: 35 ) complete image diagnostics with X-ray, CT and MRI to the time of admission were available for retrospective analysis. Image data were investigated for morphological criteria to evaluate chronological age of each sacral main fracture line bilaterally as followed: Stadium 1: bone edema in MRI STIR without any fracture evidence in CT, Stadium 2: recent fracture in CT without signs of bone callus or bony resorption, Stadium 3: fracture in healing process with blurred showing coexistence of bone apposition and resorption, Stadium 4: pseudarthrosis with wide sclerotic fracture line without signs of bone healing. 19 of these patients could not remember any associated trauma. The remainder described an inadequate rockfall event from stance or gait.

Results: In 39\% of 38 BIFS patients, chronological stadium of sacral main fracture line differed between left and right side in the individual image datasets $(n=13)$. In most cases $(\mathrm{n}=7,18 \%)$ one side showed Stadium 1 whereas the opposite side conformed to Stadium 2, indicating a short fracture sequence of $<6$ weeks. A combination of Stadium 2 and 3 was found in 6 cases (16\%). Furthermore, 23 sacra $(61 \%)$ represented the same stadium bilaterally. This fact does not approve the theory of a 2-stage fracture event. However, a short-coming sequential event $<2$ weeks can not be detected. In most of these cases recent fracture in stadium 2 was seen on both sides $(n=14,37 \%)$ followed by stadium 3 bilaterally (13\%) and stadium 1 on both sacral sides (11\%). 
Discussion: Our study results emphasize the theory of a chronological process of BIFS starting with a monolateral sacral disruption followed by a secondary contralateral fracture within a few weeks. This represents an essential difference to one-stage bilateral sacral fractures (OTA: types B3, C2.3 and C3.3) occurring within high velocity trauma. In $23 \%$ of our cases contralateral initial fracture of a BIFS could only be detected by MRI STIR images showing bony edema. In conclusion, having an obviously monolateral sacral fracture in geriatrics, a consistent MRI diagnostic is required to preclude or to confirm a contralateral lesion. This is of high importance for surgical decision-making. Therefore, an additional prophylactic stabilisation of the opposite side need to be discussed.

Fig. 1

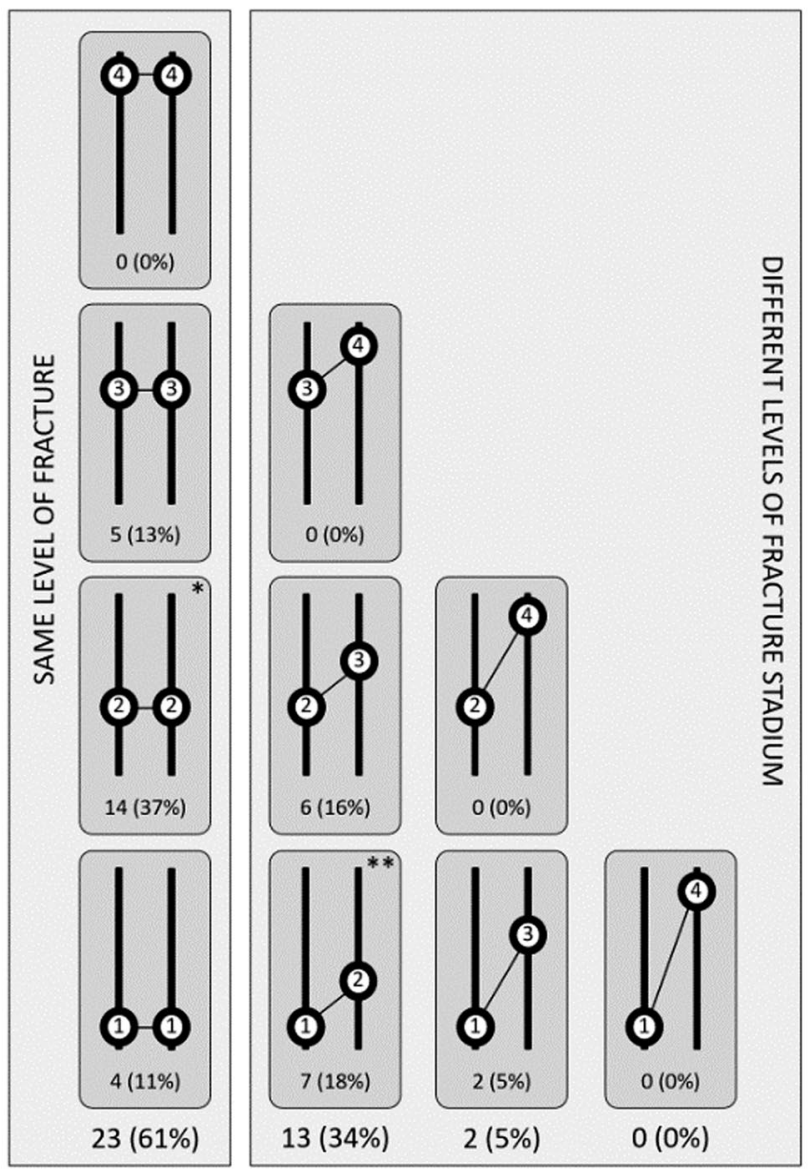

\section{55}

Effects of combined neural stem cell therapy and treadmill training on functional recovery, cyst formation and astrogliosis after cervical spinal cord injury in the rat ${ }^{*}$ A. Younsi ${ }^{1}$, M. Scherer ${ }^{1}$, L. Riemann ${ }^{1}$, G. Zheng ${ }^{1}$, T. Skutella $^{2}$, A. Unterberg ${ }^{1}$, K. Zweckberger ${ }^{1}$
${ }^{1}$ Universitätsklinikum Heidelberg, Neurochirurgische Klinik, Heidelberg, Deutschland

${ }^{2}$ Universität Heidelberg, Institut für Neuroanatomie, Heidelberg, Deutschland

Introduction: Neural precursor cell (NPC) transplantation after spinal cord injury (SCI) has shown beneficial effects on neuroregeneration in animal models. However, functional recovery may be limited due to astrogliosis and cyst formation. Treadmill training (TT) following SCI is thought to reduce these negative posttraumatic effects. We hypothesized that combining NPC transplantation and TT after SCI would improve functional recovery.

Materials and methods: $70 \mathrm{Wistar}$ rats received cervical clip-compression SCI at level C6. Animals were randomized into four treatment groups (NPC $+\mathrm{TT}$, NPC only, vehicle transplantation, sham). NPCs were injected into the spinal cord 10 days after SCI. Animals received growth factors for 7 days and were permanently immunosuppressed. Daily treadmill training was initiated $48 \mathrm{~h}$ after NPC injection. Furthermore, neurological function was assessed using Basso, Beattie and Bresnahan (BBB) score as well as CatWalk gait analysis, Gridwalk test and von Frey Filament test. After sacrifice 8 weeks post SCI, fluorescent immunohistochemical staining for astrogliosis, tissue scarring, inflammation and cyst formation was performed on frozen spinal cord sections and images were taken with a confocal laser scanning microscope. Results were compared between groups and statistically analyzed ( $p<0.05$ was considered significant).

Results: Animals who received NPC transplantation and TT recovered faster and had a significantly higher BBB score compared to NPC only and vehicle animals. While CatWalk gait analysis revealed a low Regularity Index after SCI without significant improvement over time in all injured animals, average speed was faster in NPC + TT animals compared to vehicle animals before sacrifice. Additionally, improvement of print area as well as swing speed reached a significant difference in NPC + TT animals compared to vehicle and NPC only animals. Corresponding to these results, NPC + TT animals showed significantly less stepping errors in the Gridwalk test 8 weeks after SCI. Tactile sensitivity seemed not to be pathologically altered as examined by the von Frey Filament test. Immunohistochemical analysis revealed a a significant reduction of astrogliosis and cyst size in NPC + TT animals compared to vehicle animals. Tissue scarring and inflammation were reduced in NPC + TT animals, however, a significant difference could not be observed.

Discussion: Although the BBB score showed better improvement in NPC + TT animals, the overall level of regeneration was still limited. In our SCI model, spasticity of the front paws potentially impaired neurobehavioral 
testing However, measurements like Swing- and Average Speed, less affected by spasticity, indicated superiority of combined NPC + TT treatment. These findings were supported by the Gridwalk test results. Astrogliosis and cyst size were significantly reduced when treatment with NPCs was enhanced by TT. However, tissue scarring and inflammation were not significantly affected. Collectively, our data suggests that TT may be a beneficial addition to NPC transplantation after SCI.

\section{56}

Infection prevention in spinal surgery procedures: Does the choice of antiseptic make a difference?

${ }^{*}$ C. Eder $^{1}$, P. Landowski ${ }^{2}$, G. Angerler ${ }^{3}$, J. Hahne ${ }^{4}$, S. Schildböck $^{2}$, P. Metzger ${ }^{2}$, M. Ogon ${ }^{1}$

${ }^{1}$ Orthopädisches Spital Speising, Wirbelsäulenzentrum, Wien, Österreich

${ }^{2}$ Krankenhaus Göttlicher Heiland, Abteilung für Chirurgie, Wien, Österreich

${ }^{3}$ Orthopädisches Spital Speising, Krankenhaushygiene, Wien, Österreich

${ }^{4}$ Orthopädisches Spital Speising, CEOPS - Centre of excellence for orthopaedic pain management, Wien, Österreich

Introduction: Surgicial site infections (SSI) represent a major complication of spinal surgery, with the patient's physiological skin flora being the main reservoir. The local microbiome of the back is highly specific and differs significantly from other areas of the human body. As the predominant bacteria strains are not covered by the European Standard Specifications for antiseptic evaluation, little is known regarding the specific efficacy of routinely used antiseptics for spinal surgery procedures. Aim of the presented study is to evaluate the efficacy of different antiseptic solutions in a spinal surgery specific in vitro and in vivo setting.

Materials and methods: The bacterial spectrum causing SSI after spinal surgery in our department was analysed retrospectively for 2015. Representative bacterial cultures were harvested from wound swabs and cultivated in Lysogeny Broth. PVP-Iodine $\left(\right.$ Braunol $\left.^{\circledR}\right)$, Hexetidin $\left(\right.$ Isozid $\left.^{\circledR}\right)$, Propanol/Biphenyol (Kodan forte ${ }^{\circledR}$ ) and Octenidin $\left(\right.$ Octenisept $\left.{ }^{\circledR}\right)$ were evaluated. Bacterial proliferation was monitored by measuring medium turbidity at $600 \mathrm{~nm}$ prior to disinfection, immediately and after 1 and $3 \mathrm{~h}$ to simulate the time course of a surgical procedure. Colony forming units were evaluated on agar plates. Additionally, swabs were taken in the OR from skin incision and the surgical blade after routine disinfection with Propanol/Biphenyol $(n=138)$ and after changing pre-operative skin preparation to Octenidin $(n=638)$ as a result of in vitro evaluation.

\section{Results:}

Bacterial spectrum:
Staph. epidermidis was responsible for $37 \%$ of the SSIs, followed by Propionibacterium acnes (17\%), Enterococcus faecalis (14\%), and Proteus mirabilis respectively Staph. aureus accounting for 6\% each. Staph. epidermidis, Enterococcus faecalis, Proteus mirabilis and Stap aureus could be cultivated from wound swabs and used for the subsequent evaluation.

In vitro evaluation:

The efficacy of the antiseptic solutions tested varied significantly: Octenidin demonstrated a significantly higher efficacy $(\mathrm{p}<0.01)$ in vitro and was able to completely eradicate or test bacteria except for Proteus mirabilis, which was still reduced to $1 \%$ of the initial bacterial load. In contrast, 9\% (Propanol/Biphenyol) resp. 2\% (PVP-iodine), resp. 10\% (Hexetidin) of the initial Staph epidermidis were still present after disinfection. Hexetidin showed the lowest remanent effect with Staph. epidermidis increasing to $21 \%$ of the initial bacterial load after $3 \mathrm{~h}$.

In vivo evaluation:

Following skin preparation with propanol/Biphenyol in the OR, $31 \%$ of the swabs taken resulted in positive bacterial testing. According to our in vitro results, preoperative disinfection routine was changed to Octenidin (Octeniderm $^{\circledR}$, as Octenisept ${ }^{\circledR}$ is not licensed for surgical skin preparation). Subsequently, the number of positive swabs was reduced to $22 \%$ ( $p<0.021$ ). Most of the positive swabs derived from the surgical blade and contained Staph. epidermidis or P. acnes.

Conclusion: Antisepsis does not automatically mean a total eradication of the local microbiome. Even under standardized in vitro conditions, a significant bacterial load could be detected after disinfection. The antiseptic solutions evaluated differed significantly regarding their efficacy. Octenidin demonstrated a significantly higher effectiveness on a spinal surgery specific microbioma in vitro and in vivo. While the test strains were reduced beyond the level of detection during in vitro evaluation, deeper layers of the skin, which are not reached by antiseptic treatment, may act as reservoir in vivo.

Fig. 1

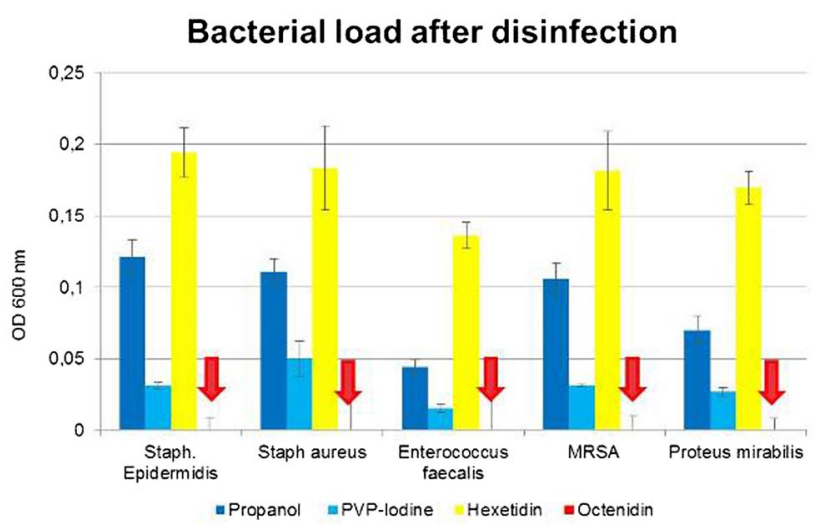


Fig. 2

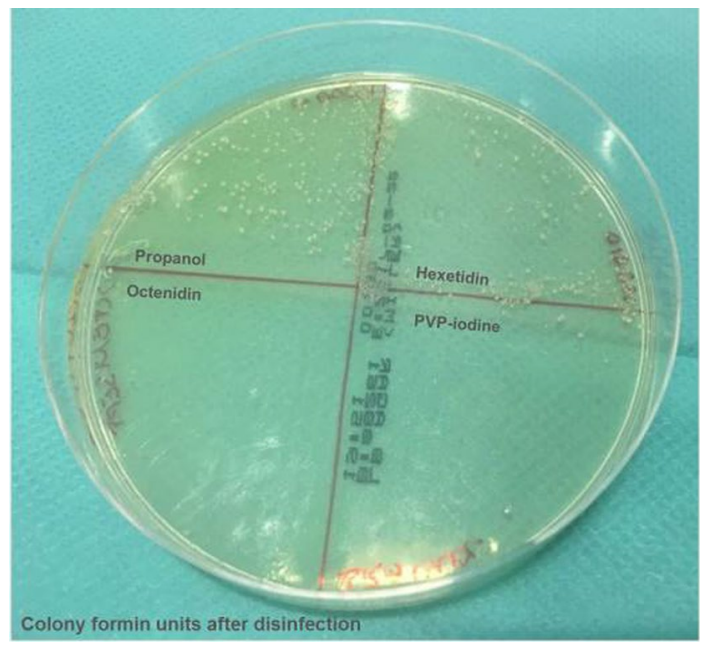

\section{57}

Comparison of three-dimensional helical axes of the cervical spine between in vitro and in vivo testing *R. Jonas ${ }^{1}$, R. Demmelmaier ${ }^{1}$, S. P. Hacker ${ }^{1}$, H. J. Wilke ${ }^{1}$ ${ }^{1}$ Universitäsklinikum Ulm, Institut für Unfallchirurgische Forschung und Biomechanik, Ulm, Deutschland

The range of motion is a well-accepted kinematic parameter for the assessment and evaluation of spinal motion. Several studies have been performed on the lumbar spine but only few qualitative data of the kinematics of the cervical spine are available which are essential for the development and success of cervical disc arthroplasty [1]. The aim of this study was to provide basic information about helical axes (HA) of human cervical spine under in vitro conditions and compare them with published in vivo data.

Six human cadaveric specimens ( 3 male and 3 female) with an average age of 48 years (range: $34-58$ years) were carefully selected. After the preparation process only the vertebrae, the intervertebral discs and the ligaments remained intact. Each specimen was divided into three motion-segments: $\mathrm{C} 2 / 3, \mathrm{C} 4 / 5$ and $\mathrm{C} 6 / 7$. For three-dimensional motion recordings 3 markers were attached to each vertebra. Afterwards all motion segments were mounted in the threedimensional spine tester. Then 3.5 full cycles of rotation about all axes: Flexion-Extension, Lateral Bending and Axial Rotation were performed by applying pure moments of $1.5 \mathrm{Nm}$ without any preload. Following the in vitro tests the three-dimensional HA and the instantaneous centers of rotation were calculated and projected into the x-ray images. Rotation analysis of all three directions revealed similar results for all six specimens. All calculated HA and instantaneous centers of rotation were in agreement with published in vivo data (Fig. 1). During Flexion/Extension the angular orientation of the HA were almost constant (Fig. 1a). In case of lateral bending the alignment of the HA was perpendicular to the articular surfaces of the facet joints (Fig. 1b). For axial rotation similar orientation of the HA could be observed. Only small deviations were present at the turning point of axial rotation (Fig. 1c).

The data gained from this study verifies cervical kinematics during in vitro testing using pure moments. Therefore in vitro experiments of cervical motion are capable of representing in vivo kinematics. As a consequence it may be assumed that the influence of muscle forces to cervical kinematics can be neglected. Only small variabilities of HA occurred due to mild degeneration artefacts within the specimens.

References: [1] Anderst, W.J. et al. (2015). Three-dimensional intervertebral kinematics in the healthy young adult cervical spine during dynamic functional loading. Journal of Biomechanics, 48(7), 1286-1293

Fig. 1

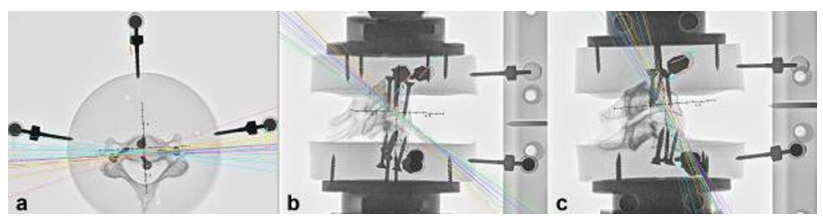

\section{58}

Temporary magnetic controlled growing rods for the treatment of severe scoliosis. A novel technique to achieve maximum curve correction and spinal height restoration - A 6 years experience.

${ }^{*}$ H. Koller ${ }^{1}$, M. Mayer ${ }^{1}$, A. Hempfing ${ }^{1}$, O. Meier ${ }^{1}$, B. Wiedenhöfer ${ }^{2}$, J. Koller ${ }^{1}$

${ }^{1}$ Schön Klinik Nürnberg Fürth, Zentrum für Wirbelsäulenund Skoliosetherapie, Fürth, Deutschland

${ }^{2}$ Werner-Wicker-Klinik, Wirbelsäulenzentrum, Bad Wildungen, Deutschland

${ }^{3}$ Universität Heidelberg, Dpt f. Wirbelsäulenchirurgie, Heidelberg, Deutschland

${ }^{4}$ Schön Klinik Lorsch, Lorsch, Deutschland

Introduction: Correction of severe thoracic curves (TC) poses distinct challenges. Recent techniques include 1.) anterior release prior to posterior correction, 2.) posterioronly correction w/ Ponte osteotomies (PO), 3.) halo-gravity traction (HGT) and posterior release prior to correction, 4.) 3 -column osteotomies (3CO) or 5.) a $360^{\circ}$-segmental release via an all-posterior approach. All these techniques have limitations due to their impact on pulmonary function, surgical risks (e.g., 3CO), and efficacy in regard to deformity correction and restoration of a balanced trunk height-to-leg length 
ratio. Staged correction of severe TC with temporary Magnetic Controlled Growing Rods (MCGR) is a novel alternate. The objectives of this study are to share our 6-years experiences with surgical and clinical outcomes in 10 cases. Methods: Prospective analysis of patient specific characteristics. In 10 pts with severe TC (TC $\geq 90^{\circ}$, TC-flexibility $\leq 30 \%)$ a staged protocol was applied including (1) internal temporary distraction with MCGR after posterior spinal release using aggressive PO, and (2) definitive correction with a segmental pedicle screw construct using neuormonitoring. Between surgeries, gradual distraction of the inserted MCGR was done once the patient was ambulatory. Preop and postop full-standing biplanar radiographs were studied for standard deformity parameters. The spinal height (trunk height) from the lowest instrumented vertebra (LIV) to $\mathrm{T} 1$ was measured btw. midpoint of upper $\mathrm{T} 1$ endplate and lower endplate of LIV. TC-correction was calculated as the scoliosis correction index (SCI: Postop TC-correction (\%) / Preop TC-Flexibility (\%)). Shoulder height (SH) and rib hump (RH) correction were also assessed as were clinical outcomes (SRS-24) and complications (acc. to Glassmann). Results: Patient age was av.16 yrs. 8 pts had AIS and 2 pts dislocating scoliosis in neurofibromatosis type 1 . In the AIS group, Lenke class. was type 3 and type 2 in each 3 pts and type 4 in 2 pts. Preop TC was av. $117^{\circ}\left(90^{\circ}-138^{\circ}\right)$ and TCflexibility was av. $19.5 \%$. Staged surgery was successfully done in all patients. After av. 14 days, the 2nd surgery was performed with removal of MCGR and corrective fusion using segmental long-head PS. The number of POs was av. $9 \pm 2$. Instrumentation was from T2 to L1-L4. Postop TC averaged $46^{\circ}$ indicating a TC-correction of $62 \pm 13 \%$ and a SCI of av. $4.3 \pm 3$. Thoracic kyphosis changed from $75^{\circ} \pm 30^{\circ}$ to $53^{\circ} \pm 14^{\circ}$ postop. At follow-up, RH-correction was av. $27 \mathrm{~mm}$ and SH-difference av. $9 \mathrm{~mm}$. Spinal height LIV-T1 increased from preop av. $289 \mathrm{~mm}$ to postop av. $380 \mathrm{~mm}$ by av. $91 \mathrm{~mm}$. No patient suffered a major complication, neurologic deficit or revision surgery. SRS-24 sum score was av. 97. One patient (NF-1), early in the case series, had suboptimal TC-correction $(<50 \%)$ due to loss of distraction by MCGR during the definitive surgical correction procedure. All patients reported they would undergo the same procedure again. 8 pts and 2 pts reported to be very satisfied/satisfied with with the outcome at a follow-up of av. 14 mos.

Conclusion: This first consecutive series on temporary internal distraction using a MCGR showed that this novel technique carries the potential to replace $\mathrm{HGT}$ or $3 \mathrm{CO}$ for the treatment of severe non-angular TC. The strategy resulted in impressive TC-corrections and restoration of trunk height of about $9 \mathrm{~cm}$, which is a unique characteristic. Postop daily external distractions centered the corrective forces at the level of greatest TC rigidity which is not possible with HGT. Results indicate that this technique carries potential to reduce indications for HGT and the need for high-risk $3 \mathrm{CO}$ for correction of severe TC. 